\title{
Networking of Vehicles - Applications, Challenges and Some Recent Results
}

\author{
Victor C.M. Leung \\ Fellow of IEEE \\ Fellow of the Engineering Institute of Canada \\ Fellow of the Canadian Academy of Engineering \\ Distinguished Lecturer of the IEEE Communications Society \\ Professional Engineer in the Province of British Columbia, Canada \\ Professor and Holder of the TELUS Mobility Research Chair in Advanced Telecommunications \\ Engineering in the Department of Electrical and Computer Engineering, University of British \\ Columbia, Vancouver, BC, Canada vleung@ece.ubc.ca
}

Recent advances in wireless communication technologies are making it possible for automobiles to be integrated into the global network. Intelligent Transportation Systems with vehicles in the loop are expected to significantly improve road safety, reduce traffic congestion and cut greenhouse gas emissions. This is made possible in the USA by Dedicated Short Range Communications (DSRC), which employs the IEEE $802.11 \mathrm{p}$ standard over the $75 \mathrm{MHz}$ of spectrum in the $5.9 \mathrm{GHz}$ band allocated by the FCC for vehicle-to-vehicle (V2V) and vehicle-to-infrastructure (V2I) communications. DSRC is expected to revolutionize road transportation by making possible many real-time safety applications. However, global deployment of DSRC is not expected to materialize in the near term due to regulatory and financial challenges. In the meantime, vehicles and their passengers are increasingly equipped with different forms of wireless networking capabilities, e.g., cellular, WiFi and WiMAX. Thus there is also a growing interest in supporting applications like infotainment, travel advisory, route planning, etc., using heterogeneous wireless networks. In this presentation, I shall describe several applications that leverage the wireless communications to put vehicles in the loop. Different applicants impose different requirements on the wireless network for data routing, transfer latency, etc. I shall review the technical challenges that need to be overcome to meet some of these requirements, and describe solutions developed in our recent research to meet these challenges. I shall conclude the presentation by discussing some future research directions. 